\section{Crystal structure of 3-(morpholin-4-yl)-1- phenyl-3-(pyridin-2-yl)propan-1-one}

\author{
F. M. Mashood Ahamed, ${ }^{\mathrm{a}}$ M. Syed Ali Padusha ${ }^{\mathrm{a}}$ and \\ B. Gunasekaran ${ }^{\text {b* }}$
}

${ }^{\text {a } P G}$ and Research Dept of Chemistry, Jamal Mohamed College (Autonomous), Tiruchirappalli, Tamil Nadu 620 020, India, and ${ }^{\mathbf{b}}$ Department of Physics \& Nano Technology, SRM University, SRM Nagar, Kattankulathur, Kancheepuram Dist, Chennai 603203 Tamil Nadu, India. *Correspondence e-mail: phdguna@gmail.com

Received 26 November 2014; accepted 30 November 2014

Edited by W. T. A. Harrison, University of Aberdeen, Scotland

In the title compound $\mathrm{C}_{18} \mathrm{H}_{20} \mathrm{~N}_{2} \mathrm{O}_{2}$, the morpholine ring adopts a chair conformation with the exocyclic $\mathrm{N}-\mathrm{C}$ bond in an equatorial orientation. The $\mathrm{N}$ atom of the morpholine ring and the $\mathrm{C}$ atom of the carbonyl group are in an anti conformation about the central $\mathrm{C}-\mathrm{C}$ bond [torsion angle $=$ $\left.-162.92(11)^{\circ}\right]$ and the dihedral angle between the planes of the benzene ring and the pyridine ring is $83.30(5)^{\circ}$. In the crystal, pairs of very weak $\mathrm{C}-\mathrm{H} \cdots \pi$ interactions link the molecules into inversion dimers.

Keywords: crystal structure; morpholin-4-yl; pyridin-2-yl; propan-1-one; biological activity.

CCDC reference: 1036843

\section{Related literature}

For background to the biological activity of morpholine derivatives, see: Panneerselvam et al. (2009); Subhashini et al. (2013); Sawant et al. (2013); Dave \& Sasaki (2006); For related structures, see: Chen et al. (2011); Meti et al. (2013);

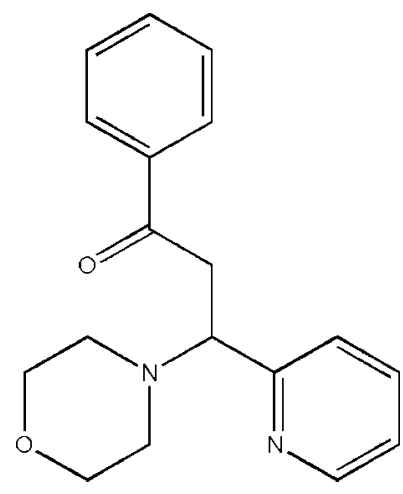

\section{Experimental}

2.1. Crystal data

$\mathrm{C}_{18} \mathrm{H}_{20} \mathrm{~N}_{2} \mathrm{O}_{2}$

$M_{r}=296.36$

Orthorhombic, $\mathrm{Pbca}$

$a=12.4554(6) \AA$

$b=8.2204(4) \AA$

$c=30.6681(17) \AA$

\subsection{Data collection}

Bruker APEXII CCD diffractometer

Absorption correction: multi-scan (SADABS; Sheldrick, 1996)

$T_{\min }=0.954, T_{\max }=0.975$

$V=3140.1(3) \AA^{3}$

$Z=8$

Mo $K \alpha$ radiation

$\mu=0.08 \mathrm{~mm}^{-1}$

$T=295 \mathrm{~K}$

$0.20 \times 0.15 \times 0.10 \mathrm{~mm}$

\subsection{Refinement}

$R\left[F^{2}>2 \sigma\left(F^{2}\right)\right]=0.043$

$w R\left(F^{2}\right)=0.111$

$S=1.03$

3812 reflections

16093 measured reflections 3812 independent reflections 2547 reflections with $I>2 \sigma(I)$

$R_{\text {int }}=0.028$

Table 1

Hydrogen-bond geometry $\left(\AA,{ }^{\circ}\right)$.

$C g 2$ is the centroid of the $\mathrm{C} 10-\mathrm{C} 14 / \mathrm{N} 1$ ring.

\begin{tabular}{lllll}
\hline$D-\mathrm{H} \cdots A$ & $D-\mathrm{H}$ & $\mathrm{H} \cdots A$ & $D \cdots A$ & $D-\mathrm{H} \cdots A$ \\
\hline $\mathrm{C} 2-\mathrm{H} 2 \cdots C g 2^{\mathrm{i}}$ & 0.93 & 2.90 & $3.780(6)$ & 159 \\
\hline
\end{tabular}

Symmetry code: (i) $-x,-y+1,-z$.

Data collection: APEX2 (Bruker, 2008); cell refinement: SAINT (Bruker, 2008); data reduction: $S A I N T$; $\operatorname{program}(\mathrm{s})$ used to solve structure: SHELXS97 (Sheldrick, 2008); program(s) used to refine structure: SHELXL97 (Sheldrick, 2008); molecular graphics: PLATON (Spek, 2009); software used to prepare material for publication: SHELXL97.

\section{Acknowledgements}

FMMA acknowledges the PG and Research Department of Chemistry and the Management of Jamal Mohamed College (Autonomous) for their kind support.

Supporting information for this paper is available from the IUCr electronic archives (Reference: HB7328).

\section{References}

Bruker (2008). APEX2 and SAINT. Bruker AXS Inc., Madison, Wisconsin, USA.

Chen, X.-Y., Zhao, M.-M., Qian, X. \& Hou, S.-G. (2011). Acta Cryst. E67, 03484 .

Dave, R. \& Sasaki, N. A. (2006). Tetrahedron Asymmetry, 17, 388-401.

Meti, G. Y., Kamble, R. R., Ravi, A. J., Arunkashi, H. K. \& Devarajegowda, H. C. (2013). Acta Cryst. E69, o129.

Panneerselvam, P., Priya, M., Gnanarupa, , Kumar, N., Ramesh, \& Saravanan, G. (2009). Indian J. Pharm. Sci. 71, 428-432.

Sawant, R. T., Stevenson, J., Odell, L. R. \& Arvidsson, P. I. (2013). Tetrahedron Asymmetry, 24, 134-141. 
Sheldrick, G. M. (1996). SADABS. University of Göttingen, Germany.

Sheldrick, G. M. (2008). Acta Cryst. A64, 112-122.

Spek, A. L. (2009). Acta Cryst. D65, 148-155.
Subhashini, N. J. P., Amanaganti, J., Boddu, L. \& Acharya Nagarjuna, P. (2013). J. Chem. Pharm. Res. 5, 140-147. 


\section{supporting information}

Acta Cryst. (2015). E71, o24-o25 [https://doi.org/10.1107/S2056989014026292]

\section{Crystal structure of 3-(morpholin-4-yl)-1-phenyl-3-(pyridin-2-yl)propan-1-one}

\section{F. M. Mashood Ahamed, M. Syed Ali Padusha and B. Gunasekaran}

\section{S1. Comment}

Morpholines are six-membered heterocycles featuring both cyclic amine and ether functional group. These compounds possess important applications in pharmaceuticals and in industries (Panneerselvam et al., 2009; Subhashini et al., 2013). Chiral morpholine derivatives have found numerous applications in asymmetric synthesis as chiral auxiliaries as well as chiral ligands (Sawant et al., 2013; Dave \& Sasaki 2006).

The geometric parameters of the title molecule (Fig. 1) agree well with reported similar structure (Chen et al., 2011; Meti et al., 2013). The morpholine (N2/O2/C15-C18)ring adopts a chair conformation $[\mathrm{Q}=0.5756(3) \AA, \Theta=179.09$ $\left.(3)^{\circ}, \varphi=332.57(5)^{\circ}\right]$. The phenyl ring makes a dihedral angles of $83.30(5)^{\circ}$ with the pyridine ring. In the crystal, a weak $\mathrm{C}-\mathrm{H} \cdots \pi$ interaction is observed.

\section{S2. Experimental}

To an ethanolic solution of acetophenone $(3.0 \mathrm{ml}, 0.025 \mathrm{~mol})$ taken in a round bottom flask, morpholine $(2.1 \mathrm{ml}, 0.025$ $\mathrm{mol})$ and pyridine-2-carboldehyde $(2.6 \mathrm{ml}, 0.025 \mathrm{~mol})$ were added. The reaction mixture was kept over a magnetic stirrer and stirred well in an ice cold condition for $3 \mathrm{hr}$. The colourless solid formed was filtered and washed several times with petroleum ether (40-60\%). The crude solid obtained was dried and recrystallized using absolute alcohol. The recrystallized product was dried over vacuum. The yield is $78 \%$ and MP is $445 \mathrm{~K}$.

\section{S3. Refinement}

$\mathrm{H}$ atoms were positioned geometrically and refined using riding model with $\mathrm{C}-\mathrm{H}=0.93 \AA$ and $U_{\text {iso }}(\mathrm{H})=1.2 \mathrm{Ueq}(\mathrm{C})$ for aromatic $\mathrm{C}-\mathrm{H}, \mathrm{C}-\mathrm{H}=0.98 \AA$ and $U_{\text {iso }}(\mathrm{H})=1.2 \mathrm{Ueq}(\mathrm{C})$ for $\mathrm{C}-\mathrm{H}, \mathrm{C}-\mathrm{H}=0.97 \AA$ and $U_{\text {iso }}(\mathrm{H})=1.2 \mathrm{Ueq}(\mathrm{C})$ for $\mathrm{C}-\mathrm{H} 2$, 


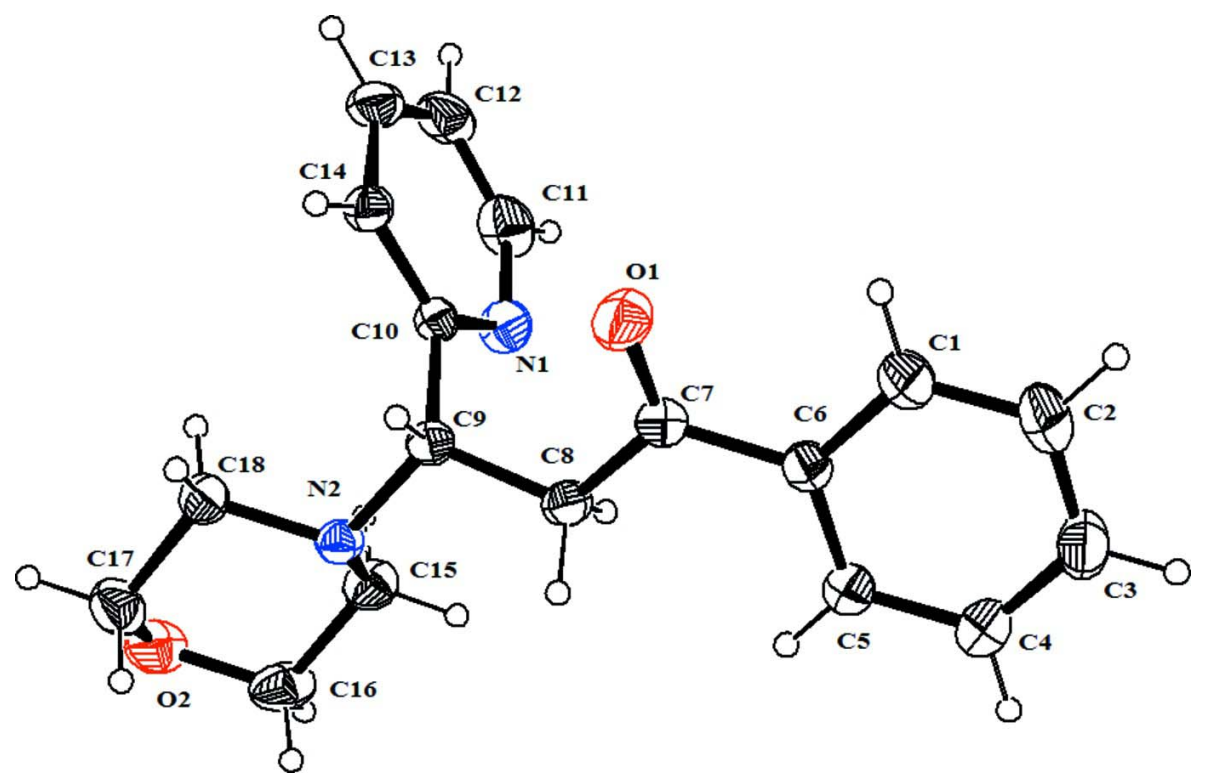

Figure 1

The molecular structure of (I), with 30\% probability displacement ellipsoids for non-H atoms.

3-(Morpholin-4-yl)-1-phenyl-3-(pyridin-2-yl)propan-1-one

Crystal data

$\mathrm{C}_{18} \mathrm{H}_{20} \mathrm{~N}_{2} \mathrm{O}_{2}$

$M_{r}=296.36$

Orthorhombic, $\mathrm{Pbca}$

Hall symbol: -P $2 \mathrm{ac} 2 \mathrm{ab}$

$a=12.4554(6) \AA$

$b=8.2204(4) \AA$

$c=30.6681(17) \AA$

$V=3140.1(3) \AA^{3}$

$Z=8$

Data collection

\section{Bruker APEXII CCD}

diffractometer

Radiation source: fine-focus sealed tube

Graphite monochromator

Detector resolution: 0 pixels $\mathrm{mm}^{-1}$

$\omega$ and $\varphi$ scans

Absorption correction: multi-scan

(SADABS; Sheldrick, 1996)

$T_{\min }=0.954, T_{\max }=0.975$

\section{Refinement}

Refinement on $F^{2}$

Least-squares matrix: full

$R\left[F^{2}>2 \sigma\left(F^{2}\right)\right]=0.043$

$w R\left(F^{2}\right)=0.111$

$S=1.03$

3812 reflections

199 parameters
$F(000)=1264$

$D_{\mathrm{x}}=1.254 \mathrm{Mg} \mathrm{m}^{-3}$

Mo $K \alpha$ radiation, $\lambda=0.71073 \AA$

Cell parameters from 3812 reflections

$\theta=1.3-28.4^{\circ}$

$\mu=0.08 \mathrm{~mm}^{-1}$

$T=295 \mathrm{~K}$

Block, colourless

$0.20 \times 0.15 \times 0.10 \mathrm{~mm}$

16093 measured reflections

3812 independent reflections

2547 reflections with $I>2 \sigma(I)$

$R_{\text {int }}=0.028$

$\theta_{\text {max }}=28.4^{\circ}, \theta_{\min }=1.3^{\circ}$

$h=-15 \rightarrow 15$

$k=-10 \rightarrow 10$

$l=-35 \rightarrow 40$

0 restraints

Primary atom site location: structure-invariant direct methods

Secondary atom site location: difference Fourier map

Hydrogen site location: inferred from neighbouring sites 
H-atom parameters constrained

$w=1 /\left[\sigma^{2}\left(F_{\mathrm{o}}^{2}\right)+(0.0416 P)^{2}+0.6298 P\right]$

where $P=\left(F_{\mathrm{o}}^{2}+2 F_{\mathrm{c}}^{2}\right) / 3$

$$
\begin{aligned}
& (\Delta / \sigma)_{\max }<0.001 \\
& \Delta \rho_{\max }=0.14 \mathrm{e} \AA^{-3} \\
& \Delta \rho_{\min }=-0.16 \mathrm{e} \AA^{-3}
\end{aligned}
$$

\begin{tabular}{|c|c|c|c|c|}
\hline & $x$ & $y$ & $z$ & $U_{\text {iso }} * / U_{\text {eq }}$ \\
\hline $\mathrm{C} 1$ & $0.42802(13)$ & $-0.1732(2)$ & $0.02138(5)$ & $0.0604(4)$ \\
\hline H1 & 0.5020 & -0.1642 & 0.0182 & $0.073 *$ \\
\hline $\mathrm{C} 2$ & $0.36782(16)$ & $-0.2413(2)$ & $-0.01152(6)$ & $0.0746(5)$ \\
\hline $\mathrm{H} 2$ & 0.4013 & -0.2768 & -0.0369 & $0.089 *$ \\
\hline $\mathrm{C} 3$ & $0.25900(16)$ & $-0.2571(2)$ & $-0.00707(5)$ & $0.0680(5)$ \\
\hline H3 & 0.2186 & -0.3050 & -0.0291 & $0.082 *$ \\
\hline $\mathrm{C} 4$ & $0.21022(13)$ & $-0.2020(2)$ & $0.02998(6)$ & $0.0653(5)$ \\
\hline $\mathrm{H} 4$ & 0.1362 & -0.2121 & 0.0330 & $0.078^{*}$ \\
\hline $\mathrm{C} 5$ & $0.26935(12)$ & $-0.13170(19)$ & $0.06292(5)$ & $0.0529(4)$ \\
\hline H5 & 0.2350 & -0.0932 & 0.0878 & $0.063 *$ \\
\hline C6 & $0.37980(11)$ & $-0.11808(15)$ & 0.05909 (4) & $0.0412(3)$ \\
\hline $\mathrm{C} 7$ & $0.44770(10)$ & $-0.04735(16)$ & $0.09421(4)$ & $0.0417(3)$ \\
\hline $\mathrm{C} 8$ & $0.39381(10)$ & 0.03707 (17) & $0.13181(4)$ & $0.0429(3)$ \\
\hline $\mathrm{H} 8 \mathrm{~A}$ & 0.3510 & -0.0418 & 0.1477 & $0.051 *$ \\
\hline H8B & 0.3453 & 0.1190 & 0.1204 & $0.051 *$ \\
\hline C9 & $0.47105(9)$ & $0.11758(15)$ & $0.16326(4)$ & $0.0364(3)$ \\
\hline H9 & 0.5269 & 0.0372 & 0.1697 & $0.044^{*}$ \\
\hline $\mathrm{C} 10$ & $0.52724(10)$ & $0.26260(15)$ & $0.14299(4)$ & $0.0368(3)$ \\
\hline C11 & $0.51464(16)$ & $0.4990(2)$ & $0.10454(6)$ & $0.0666(5)$ \\
\hline H11 & 0.4726 & 0.5746 & 0.0898 & $0.080 *$ \\
\hline $\mathrm{C} 12$ & $0.62216(16)$ & $0.5287(2)$ & $0.10752(6)$ & $0.0678(5)$ \\
\hline H12 & 0.6520 & 0.6215 & 0.0951 & $0.081^{*}$ \\
\hline C13 & $0.68473(13)$ & $0.4190(2)$ & $0.12904(5)$ & $0.0610(5)$ \\
\hline H13 & 0.7585 & 0.4344 & 0.1313 & $0.073^{*}$ \\
\hline $\mathrm{C} 14$ & $0.63651(11)$ & $0.28526(18)$ & $0.14735(5)$ & $0.0452(3)$ \\
\hline H14 & 0.6775 & 0.2099 & 0.1627 & $0.054 *$ \\
\hline $\mathrm{C} 15$ & $0.33073(11)$ & $0.2736(2)$ & $0.20287(5)$ & $0.0532(4)$ \\
\hline H15A & 0.2787 & 0.2424 & 0.1809 & $0.064 *$ \\
\hline H15B & 0.3602 & 0.3787 & 0.1949 & $0.064 *$ \\
\hline $\mathrm{C} 16$ & $0.27665(12)$ & $0.2851(2)$ & $0.24674(6)$ & $0.0662(5)$ \\
\hline H16A & 0.2195 & 0.3651 & 0.2453 & $0.079 *$ \\
\hline H16B & 0.2447 & 0.1808 & 0.2539 & $0.079 *$ \\
\hline $\mathrm{C} 17$ & $0.43388(14)$ & $0.2128(2)$ & $0.28202(5)$ & $0.0632(4)$ \\
\hline H17A & 0.4040 & 0.1078 & 0.2898 & $0.076^{*}$ \\
\hline H17B & 0.4846 & 0.2440 & 0.3045 & $0.076^{*}$ \\
\hline C18 & $0.49150(11)$ & $0.19882(18)$ & $0.23921(4)$ & $0.0462(3)$ \\
\hline H18A & 0.5247 & 0.3021 & 0.2320 & $0.055^{*}$ \\
\hline H18B & 0.5477 & 0.1176 & 0.2415 & $0.055^{*}$ \\
\hline N1 & $0.46563(10)$ & $0.36867(15)$ & $0.12143(4)$ & $0.0526(3)$ \\
\hline N2 & $0.41661(8)$ & $0.15290(13)$ & $0.20488(4)$ & $0.0394(3)$ \\
\hline $\mathrm{O} 1$ & $0.54495(8)$ & $-0.05833(15)$ & $0.09246(4)$ & $0.0655(3)$ \\
\hline
\end{tabular}

Fractional atomic coordinates and isotropic or equivalent isotropic displacement parameters $\left(\AA^{2}\right)$ 


$\begin{array}{lllll}\mathrm{O} 2 & 0.35010(10) & 0.32957(14) & 0.27995(4) & 0.0678(3)\end{array}$

Atomic displacement parameters $\left(\AA^{2}\right)$

\begin{tabular}{lllllll}
\hline & $U^{11}$ & $U^{22}$ & $U^{33}$ & $U^{12}$ & $U^{13}$ & $U^{23}$ \\
\hline C1 & $0.0564(9)$ & $0.0742(11)$ & $0.0506(9)$ & $-0.0007(8)$ & $0.0083(8)$ & $-0.0126(8)$ \\
C2 & $0.0842(13)$ & $0.0925(14)$ & $0.0470(10)$ & $-0.0019(11)$ & $0.0067(9)$ & $-0.0220(9)$ \\
C3 & $0.0815(12)$ & $0.0728(11)$ & $0.0497(9)$ & $-0.0123(10)$ & $-0.0134(9)$ & $-0.0073(9)$ \\
C4 & $0.0567(9)$ & $0.0784(12)$ & $0.0607(10)$ & $-0.0166(9)$ & $-0.0049(8)$ & $-0.0085(9)$ \\
C5 & $0.0495(9)$ & $0.0615(9)$ & $0.0477(8)$ & $-0.0098(7)$ & $0.0030(7)$ & $-0.0074(7)$ \\
C6 & $0.0473(7)$ & $0.0366(7)$ & $0.0399(7)$ & $-0.0009(6)$ & $0.0012(6)$ & $0.0008(6)$ \\
C7 & $0.0410(7)$ & $0.0392(7)$ & $0.0449(8)$ & $0.0005(6)$ & $0.0027(6)$ & $0.0003(6)$ \\
C8 & $0.0379(7)$ & $0.0446(7)$ & $0.0461(8)$ & $-0.0049(6)$ & $0.0040(6)$ & $-0.0039(6)$ \\
C9 & $0.0328(6)$ & $0.0363(7)$ & $0.0402(7)$ & $0.0024(5)$ & $0.0011(5)$ & $0.0002(6)$ \\
C10 & $0.0376(7)$ & $0.0382(7)$ & $0.0346(6)$ & $-0.0004(5)$ & $0.0020(5)$ & $-0.0025(5)$ \\
C11 & $0.0910(13)$ & $0.0498(9)$ & $0.0589(10)$ & $-0.0004(9)$ & $-0.0032(9)$ & $0.0166(8)$ \\
C12 & $0.0926(13)$ & $0.0549(10)$ & $0.0559(10)$ & $-0.0279(10)$ & $0.0183(9)$ & $0.0017(8)$ \\
C13 & $0.0555(9)$ & $0.0663(10)$ & $0.0613(10)$ & $-0.0229(8)$ & $0.0155(8)$ & $-0.0162(9)$ \\
C14 & $0.0389(7)$ & $0.0512(8)$ & $0.0456(8)$ & $-0.0027(6)$ & $0.0030(6)$ & $-0.0067(7)$ \\
C15 & $0.0404(7)$ & $0.0569(9)$ & $0.0624(10)$ & $0.0062(7)$ & $0.0048(7)$ & $-0.0090(8)$ \\
C16 & $0.0500(9)$ & $0.0641(10)$ & $0.0844(12)$ & $-0.0057(8)$ & $0.0237(9)$ & $-0.0198(9)$ \\
C17 & $0.0810(11)$ & $0.0612(10)$ & $0.0473(9)$ & $-0.0109(9)$ & $0.0080(8)$ & $-0.0065(8)$ \\
C18 & $0.0483(8)$ & $0.0451(8)$ & $0.0453(8)$ & $-0.0029(7)$ & $-0.0002(6)$ & $-0.0014(6)$ \\
N1 & $0.0530(7)$ & $0.0496(7)$ & $0.0553(7)$ & $0.0018(6)$ & $-0.0067(6)$ & $0.0125(6)$ \\
N2 & $0.0355(5)$ & $0.0409(6)$ & $0.0419(6)$ & $-0.0012(5)$ & $0.0040(5)$ & $-0.0024(5)$ \\
O1 & $0.0412(6)$ & $0.0847(9)$ & $0.0707(8)$ & $0.0064(5)$ & $0.0016(5)$ & $-0.0247(6)$ \\
O2 & $0.0733(7)$ & $0.0648(7)$ & $0.0654(7)$ & $-0.0114(6)$ & $0.0215(6)$ & $-0.0235(6)$ \\
& & & & & & \\
& & & & & &
\end{tabular}

Geometric parameters $\left(\AA,^{\circ}\right)$

\begin{tabular}{llll}
\hline $\mathrm{C} 1-\mathrm{C} 2$ & $1.376(2)$ & $\mathrm{C} 11-\mathrm{N} 1$ & $1.338(2)$ \\
$\mathrm{C} 1-\mathrm{C} 6$ & $1.380(2)$ & $\mathrm{C} 11-\mathrm{C} 12$ & $1.364(3)$ \\
$\mathrm{C} 1-\mathrm{H} 1$ & 0.9300 & $\mathrm{C} 11-\mathrm{H} 11$ & 0.9300 \\
$\mathrm{C} 2-\mathrm{C} 3$ & $1.368(3)$ & $\mathrm{C} 12-\mathrm{C} 13$ & $1.362(2)$ \\
$\mathrm{C} 2-\mathrm{H} 2$ & 0.9300 & $\mathrm{C} 12-\mathrm{H} 12$ & 0.9300 \\
$\mathrm{C} 3-\mathrm{C} 4$ & $1.366(2)$ & $\mathrm{C} 13-\mathrm{C} 14$ & $1.373(2)$ \\
$\mathrm{C} 3-\mathrm{H} 3$ & 0.9300 & $\mathrm{C} 13-\mathrm{H} 13$ & 0.9300 \\
$\mathrm{C} 4-\mathrm{C} 5$ & $1.377(2)$ & $\mathrm{C} 14-\mathrm{H} 14$ & 0.9300 \\
$\mathrm{C} 4-\mathrm{H} 4$ & 0.9300 & $\mathrm{C} 15-\mathrm{N} 2$ & $1.4604(17)$ \\
$\mathrm{C} 5-\mathrm{C} 6$ & $1.3852(19)$ & $\mathrm{C} 15-\mathrm{C} 16$ & $1.508(2)$ \\
$\mathrm{C} 5-\mathrm{H} 5$ & 0.9300 & $\mathrm{C} 15-\mathrm{H} 15 \mathrm{~A}$ & 0.9700 \\
$\mathrm{C} 6-\mathrm{C} 7$ & $1.4878(19)$ & $\mathrm{C} 15-\mathrm{H} 15 \mathrm{~B}$ & 0.9700 \\
$\mathrm{C} 7-\mathrm{O} 1$ & $1.2159(15)$ & $\mathrm{C} 16-\mathrm{O} 2$ & $1.417(2)$ \\
$\mathrm{C} 7-\mathrm{C} 8$ & $1.5039(19)$ & $\mathrm{C} 16-\mathrm{H} 16 \mathrm{~A}$ & 0.9700 \\
$\mathrm{C} 8-\mathrm{C} 9$ & $1.5146(18)$ & $\mathrm{C} 16-\mathrm{H} 16 \mathrm{~B}$ & 0.9700 \\
$\mathrm{C} 8-\mathrm{H} 8 \mathrm{~A}$ & 0.9700 & $\mathrm{C} 17-\mathrm{O} 2$ & $1.419(2)$ \\
$\mathrm{C} 8-\mathrm{H} 8 \mathrm{~B}$ & 0.9700 & $\mathrm{C} 17-\mathrm{C} 18$ & $1.500(2)$ \\
$\mathrm{C} 9-\mathrm{N} 2$ & $1.4741(16)$ & $\mathrm{C} 17-\mathrm{H} 17 \mathrm{~A}$ & 0.9700
\end{tabular}




\begin{tabular}{|c|c|c|c|}
\hline $\mathrm{C} 9-\mathrm{C} 10$ & $1.5157(18)$ & C17-H17B & 0.9700 \\
\hline $\mathrm{C} 9-\mathrm{H} 9$ & 0.9800 & $\mathrm{C} 18-\mathrm{N} 2$ & $1.4565(17)$ \\
\hline $\mathrm{C} 10-\mathrm{N} 1$ & $1.3365(17)$ & $\mathrm{C} 18-\mathrm{H} 18 \mathrm{~A}$ & 0.9700 \\
\hline $\mathrm{C} 10-\mathrm{C} 14$ & $1.3803(17)$ & $\mathrm{C} 18-\mathrm{H} 18 \mathrm{~B}$ & 0.9700 \\
\hline $\mathrm{C} 2-\mathrm{C} 1-\mathrm{C} 6$ & $120.73(15)$ & $\mathrm{C} 13-\mathrm{C} 12-\mathrm{C} 11$ & $118.42(15)$ \\
\hline $\mathrm{C} 2-\mathrm{C} 1-\mathrm{H} 1$ & 119.6 & $\mathrm{C} 13-\mathrm{C} 12-\mathrm{H} 12$ & 120.8 \\
\hline $\mathrm{C} 6-\mathrm{C} 1-\mathrm{H} 1$ & 119.6 & $\mathrm{C} 11-\mathrm{C} 12-\mathrm{H} 12$ & 120.8 \\
\hline $\mathrm{C} 3-\mathrm{C} 2-\mathrm{C} 1$ & $120.36(16)$ & $\mathrm{C} 12-\mathrm{C} 13-\mathrm{C} 14$ & $118.55(15)$ \\
\hline $\mathrm{C} 3-\mathrm{C} 2-\mathrm{H} 2$ & 119.8 & $\mathrm{C} 12-\mathrm{C} 13-\mathrm{H} 13$ & 120.7 \\
\hline $\mathrm{C} 1-\mathrm{C} 2-\mathrm{H} 2$ & 119.8 & $\mathrm{C} 14-\mathrm{C} 13-\mathrm{H} 13$ & 120.7 \\
\hline $\mathrm{C} 4-\mathrm{C} 3-\mathrm{C} 2$ & $119.47(16)$ & $\mathrm{C} 13-\mathrm{C} 14-\mathrm{C} 10$ & $119.99(15)$ \\
\hline $\mathrm{C} 4-\mathrm{C} 3-\mathrm{H} 3$ & 120.3 & $\mathrm{C} 13-\mathrm{C} 14-\mathrm{H} 14$ & 120.0 \\
\hline $\mathrm{C} 2-\mathrm{C} 3-\mathrm{H} 3$ & 120.3 & $\mathrm{C} 10-\mathrm{C} 14-\mathrm{H} 14$ & 120.0 \\
\hline $\mathrm{C} 3-\mathrm{C} 4-\mathrm{C} 5$ & $120.76(15)$ & $\mathrm{N} 2-\mathrm{C} 15-\mathrm{C} 16$ & $109.39(13)$ \\
\hline $\mathrm{C} 3-\mathrm{C} 4-\mathrm{H} 4$ & 119.6 & $\mathrm{~N} 2-\mathrm{C} 15-\mathrm{H} 15 \mathrm{~A}$ & 109.8 \\
\hline $\mathrm{C} 5-\mathrm{C} 4-\mathrm{H} 4$ & 119.6 & $\mathrm{C} 16-\mathrm{C} 15-\mathrm{H} 15 \mathrm{~A}$ & 109.8 \\
\hline $\mathrm{C} 4-\mathrm{C} 5-\mathrm{C} 6$ & $120.18(14)$ & $\mathrm{N} 2-\mathrm{C} 15-\mathrm{H} 15 \mathrm{~B}$ & 109.8 \\
\hline $\mathrm{C} 4-\mathrm{C} 5-\mathrm{H} 5$ & 119.9 & $\mathrm{C} 16-\mathrm{C} 15-\mathrm{H} 15 \mathrm{~B}$ & 109.8 \\
\hline $\mathrm{C} 6-\mathrm{C} 5-\mathrm{H} 5$ & 119.9 & $\mathrm{H} 15 \mathrm{~A}-\mathrm{C} 15-\mathrm{H} 15 \mathrm{~B}$ & 108.2 \\
\hline $\mathrm{C} 1-\mathrm{C} 6-\mathrm{C} 5$ & $118.48(13)$ & $\mathrm{O} 2-\mathrm{C} 16-\mathrm{C} 15$ & $111.65(12)$ \\
\hline $\mathrm{C} 1-\mathrm{C} 6-\mathrm{C} 7$ & $119.19(12)$ & $\mathrm{O} 2-\mathrm{C} 16-\mathrm{H} 16 \mathrm{~A}$ & 109.3 \\
\hline $\mathrm{C} 5-\mathrm{C} 6-\mathrm{C} 7$ & $122.33(12)$ & $\mathrm{C} 15-\mathrm{C} 16-\mathrm{H} 16 \mathrm{~A}$ & 109.3 \\
\hline $\mathrm{O} 1-\mathrm{C} 7-\mathrm{C} 6$ & $120.35(13)$ & $\mathrm{O} 2-\mathrm{C} 16-\mathrm{H} 16 \mathrm{~B}$ & 109.3 \\
\hline $\mathrm{O} 1-\mathrm{C} 7-\mathrm{C} 8$ & $120.85(12)$ & $\mathrm{C} 15-\mathrm{C} 16-\mathrm{H} 16 \mathrm{~B}$ & 109.3 \\
\hline $\mathrm{C} 6-\mathrm{C} 7-\mathrm{C} 8$ & $118.80(11)$ & $\mathrm{H} 16 \mathrm{~A}-\mathrm{C} 16-\mathrm{H} 16 \mathrm{~B}$ & 108.0 \\
\hline $\mathrm{C} 7-\mathrm{C} 8-\mathrm{C} 9$ & $113.98(10)$ & $\mathrm{O} 2-\mathrm{C} 17-\mathrm{C} 18$ & $111.37(13)$ \\
\hline $\mathrm{C} 7-\mathrm{C} 8-\mathrm{H} 8 \mathrm{~A}$ & 108.8 & $\mathrm{O} 2-\mathrm{C} 17-\mathrm{H} 17 \mathrm{~A}$ & 109.4 \\
\hline $\mathrm{C} 9-\mathrm{C} 8-\mathrm{H} 8 \mathrm{~A}$ & 108.8 & $\mathrm{C} 18-\mathrm{C} 17-\mathrm{H} 17 \mathrm{~A}$ & 109.4 \\
\hline $\mathrm{C} 7-\mathrm{C} 8-\mathrm{H} 8 \mathrm{~B}$ & 108.8 & $\mathrm{O} 2-\mathrm{C} 17-\mathrm{H} 17 \mathrm{~B}$ & 109.4 \\
\hline $\mathrm{C} 9-\mathrm{C} 8-\mathrm{H} 8 \mathrm{~B}$ & 108.8 & $\mathrm{C} 18-\mathrm{C} 17-\mathrm{H} 17 \mathrm{~B}$ & 109.4 \\
\hline $\mathrm{H} 8 \mathrm{~A}-\mathrm{C} 8-\mathrm{H} 8 \mathrm{~B}$ & 107.7 & $\mathrm{H} 17 \mathrm{~A}-\mathrm{C} 17-\mathrm{H} 17 \mathrm{~B}$ & 108.0 \\
\hline $\mathrm{N} 2-\mathrm{C} 9-\mathrm{C} 8$ & $110.20(10)$ & $\mathrm{N} 2-\mathrm{C} 18-\mathrm{C} 17$ & $110.24(12)$ \\
\hline $\mathrm{N} 2-\mathrm{C} 9-\mathrm{C} 10$ & $114.37(10)$ & $\mathrm{N} 2-\mathrm{C} 18-\mathrm{H} 18 \mathrm{~A}$ & 109.6 \\
\hline $\mathrm{C} 8-\mathrm{C} 9-\mathrm{C} 10$ & $112.07(11)$ & $\mathrm{C} 17-\mathrm{C} 18-\mathrm{H} 18 \mathrm{~A}$ & 109.6 \\
\hline $\mathrm{N} 2-\mathrm{C} 9-\mathrm{H} 9$ & 106.6 & $\mathrm{~N} 2-\mathrm{C} 18-\mathrm{H} 18 \mathrm{~B}$ & 109.6 \\
\hline $\mathrm{C} 8-\mathrm{C} 9-\mathrm{H} 9$ & 106.6 & $\mathrm{C} 17-\mathrm{C} 18-\mathrm{H} 18 \mathrm{~B}$ & 109.6 \\
\hline $\mathrm{C} 10-\mathrm{C} 9-\mathrm{H} 9$ & 106.6 & $\mathrm{H} 18 \mathrm{~A}-\mathrm{C} 18-\mathrm{H} 18 \mathrm{~B}$ & 108.1 \\
\hline $\mathrm{N} 1-\mathrm{C} 10-\mathrm{C} 14$ & $121.74(13)$ & $\mathrm{C} 10-\mathrm{N} 1-\mathrm{C} 11$ & $116.89(13)$ \\
\hline $\mathrm{N} 1-\mathrm{C} 10-\mathrm{C} 9$ & $116.80(11)$ & $\mathrm{C} 18-\mathrm{N} 2-\mathrm{C} 15$ & $108.87(11)$ \\
\hline $\mathrm{C} 14-\mathrm{C} 10-\mathrm{C} 9$ & $121.45(12)$ & $\mathrm{C} 18-\mathrm{N} 2-\mathrm{C} 9$ & $112.48(10)$ \\
\hline $\mathrm{N} 1-\mathrm{C} 11-\mathrm{C} 12$ & $124.40(17)$ & $\mathrm{C} 15-\mathrm{N} 2-\mathrm{C} 9$ & $115.74(11)$ \\
\hline $\mathrm{N} 1-\mathrm{C} 11-\mathrm{H} 11$ & 117.8 & $\mathrm{C} 16-\mathrm{O} 2-\mathrm{C} 17$ & $109.40(12)$ \\
\hline $\mathrm{C} 12-\mathrm{C} 11-\mathrm{H} 11$ & 117.8 & & \\
\hline $\mathrm{C} 6-\mathrm{C} 1-\mathrm{C} 2-\mathrm{C} 3$ & $-0.8(3)$ & $\mathrm{N} 1-\mathrm{C} 11-\mathrm{C} 12-\mathrm{C} 13$ & $0.0(3)$ \\
\hline $\mathrm{C} 1-\mathrm{C} 2-\mathrm{C} 3-\mathrm{C} 4$ & $1.2(3)$ & $\mathrm{C} 11-\mathrm{C} 12-\mathrm{C} 13-\mathrm{C} 14$ & $-1.1(2)$ \\
\hline $\mathrm{C} 2-\mathrm{C} 3-\mathrm{C} 4-\mathrm{C} 5$ & $-0.3(3)$ & $\mathrm{C} 12-\mathrm{C} 13-\mathrm{C} 14-\mathrm{C} 10$ & $1.3(2)$ \\
\hline
\end{tabular}




$\mathrm{C} 3-\mathrm{C} 4-\mathrm{C} 5-\mathrm{C} 6$
$\mathrm{C} 2-\mathrm{C} 1-\mathrm{C} 6-\mathrm{C} 5$
$\mathrm{C} 2-\mathrm{C} 1-\mathrm{C} 6-\mathrm{C} 7$
$\mathrm{C} 4-\mathrm{C} 5-\mathrm{C} 6-\mathrm{C} 1$
$\mathrm{C} 4-\mathrm{C} 5-\mathrm{C} 6-\mathrm{C} 7$
$\mathrm{C} 1-\mathrm{C} 6-\mathrm{C} 7-\mathrm{O} 1$
$\mathrm{C} 5-\mathrm{C} 6-\mathrm{C} 7-\mathrm{O} 1$
$\mathrm{C} 1-\mathrm{C} 6-\mathrm{C} 7-\mathrm{C} 8$
$\mathrm{C} 5-\mathrm{C} 6-\mathrm{C} 7-\mathrm{C} 8$
$\mathrm{O} 1-\mathrm{C} 7-\mathrm{C} 8-\mathrm{C} 9$
$\mathrm{C} 6-\mathrm{C} 7-\mathrm{C} 8-\mathrm{C} 9$
$\mathrm{C} 7-\mathrm{C} 8-\mathrm{C} 9-\mathrm{N} 2$
$\mathrm{C} 7-\mathrm{C} 8-\mathrm{C} 9-\mathrm{C} 10$
$\mathrm{~N} 2-\mathrm{C} 9-\mathrm{C} 10-\mathrm{N} 1$
$\mathrm{C} 8-\mathrm{C} 9-\mathrm{C} 10-\mathrm{N} 1$
$\mathrm{~N} 2-\mathrm{C} 9-\mathrm{C} 10-\mathrm{C} 14$
$\mathrm{C} 8-\mathrm{C} 9-\mathrm{C} 10-\mathrm{C} 14$

$$
-0.9(3)
$$$$
-0.5 \text { (2) }
$$

$179.11(16)$

$1.3(2)$

$-178.26(14)$

$-10.1(2)$

$169.48(15)$

$170.08(13)$

$-10.3(2)$

$5.6(2)$

$-174.59(11)$

$-162.92(11)$

$68.50(15)$

$-79.35(15)$

$47.00(15)$

99.47 (14)

-134.18 (13)

$\begin{array}{ll}\mathrm{N} 1-\mathrm{C} 10-\mathrm{C} 14-\mathrm{C} 13 & -0.4(2) \\ \mathrm{C} 9-\mathrm{C} 10-\mathrm{C} 14-\mathrm{C} 13 & -179.19(13) \\ \mathrm{N} 2-\mathrm{C} 15-\mathrm{C} 16-\mathrm{O} 2 & 59.10(17) \\ \mathrm{O} 2-\mathrm{C} 17-\mathrm{C} 18-\mathrm{N} 2 & -58.71(16) \\ \mathrm{C} 14-\mathrm{C} 10-\mathrm{N} 1-\mathrm{C} 11 & -0.6(2) \\ \mathrm{C} 9-\mathrm{C} 10-\mathrm{N} 1-\mathrm{C} 11 & 178.23(13) \\ \mathrm{C} 12-\mathrm{C} 11-\mathrm{N} 1-\mathrm{C} 10 & 0.8(3) \\ \mathrm{C} 17-\mathrm{C} 18-\mathrm{N} 2-\mathrm{C} 15 & 57.48(15) \\ \mathrm{C} 17-\mathrm{C} 18-\mathrm{N} 2-\mathrm{C} 9 & -172.87(12) \\ \mathrm{C} 16-\mathrm{C} 15-\mathrm{N} 2-\mathrm{C} 18 & -57.27(15) \\ \mathrm{C} 16-\mathrm{C} 15-\mathrm{N} 2-\mathrm{C} 9 & 174.89(11) \\ \mathrm{C} 8-\mathrm{C} 9-\mathrm{N} 2-\mathrm{C} 18 & 168.06(11) \\ \mathrm{C} 10-\mathrm{C} 9-\mathrm{N} 2-\mathrm{C} 18 & -64.62(14) \\ \mathrm{C} 8-\mathrm{C} 9-\mathrm{N} 2-\mathrm{C} 15 & -65.92(14) \\ \mathrm{C} 10-\mathrm{C} 9-\mathrm{N} 2-\mathrm{C} 15 & 61.40(14) \\ \mathrm{C} 15-\mathrm{C} 16-\mathrm{O} 2-\mathrm{C} 17 & -58.86(18) \\ \mathrm{C} 18-\mathrm{C} 17-\mathrm{O} 2-\mathrm{C} 16 & 58.37(16) \\ \end{array}$

$\mathrm{N} 1-\mathrm{C} 10-\mathrm{C} 14-\mathrm{C} 13$

$-0.4(2)$

$59.10(17)$

$-58.71(16)$

$-0.6(2)$

$178.23(13)$

$0.8(3)$

$57.48(15)$

$-172.87(12)$

$-57.27(15)$

$174.89(11)$

$168.06(11)$

$-64.62(14)$

$61.40(14)$

$58.37(16)$

Hydrogen-bond geometry $\left(\AA,{ }^{\circ}\right)$

$\mathrm{Cg} 2$ is the centroid of the $\mathrm{C} 10-\mathrm{C} 14 / \mathrm{N} 1$ ring.

\begin{tabular}{lllll}
\hline$D-\mathrm{H} \cdots A$ & $D-\mathrm{H}$ & $\mathrm{H} \cdots A$ & $D \cdots A$ & $D-\mathrm{H} \cdots A$ \\
\hline $\mathrm{C} 2-\mathrm{H} 2 \cdots C g 2^{\mathrm{i}}$ & 0.93 & 2.90 & $3.780(6)$ & 159 \\
\hline
\end{tabular}

Symmetry code: (i) $-x,-y+1,-z$. 